\title{
CONSIDERAZIONI SULLA PIRATERIA E SULLA CORSA MUSULMANA E CRISTIANA ALL'EPOCA DELLA CONQUISTA PORTOGHESE DI CEUTA
}

(1415)

\author{
ANNA UNALI \\ Universitd di Roma "La Sapienza" \\ (Italia)
}

Nei secoli finali del Bassomedioevo il Mediterraneo occidentale si trovava in una situazione di contl ittualita permanente non solo per l'ostilità episodica degli stati cristiani e musulmani ma soprattutto a causa dell'attivita predatoria di corsari e pirati. Il pericolo insito nei viaggi per mare in quell'area si accentud da parte cristiana specialmente durante la seconda metà del Trecento e nei primi decenni del ' 400 nel momento in cui vi fu come elemento caratterizzante una concentrazione di interessi economici nella zona marittima dello Stretto di Gibilterra.

La ragione dell'importanza di tale polo mediterraneo si ritrova nella sua posizione geografica e nella concomitanza di obiettivi perseguiti dagli stati di entrambe le sponde del Mediterraneo ${ }^{1}$. Tra di essi si devono annoverare i naturali fruitori di quel braccio di mare e cioé la Casiglia, il regno di Granada e il Magreb al-Aksá (dell'estremo Occidente), ma anche, per la loro consolidata direttrice commerciale verso Occidente, l'Aragona e Genova.

\footnotetext{
'Valentim Femandes a proposito di Ceuta quale importante centro commerciale e scalo mediterranco dice: " Esta foy huma das principaes cidades no tempo dos mouros assy em edificios coino $\mathrm{cm}$ riquezas e nobrezas e mercadorias... E estava $\mathrm{cm}$ tanta prosperidade que quantos navios passassem pello dito estreito quer de levante ou poente aviam de amaynar as velas ..."; efr. O Manuscrito Valention Fernandes. Ceula e a sua costa, Leitura e Revisão das provos pelo academico A. Baiño, Lisboa, 1940, p. 33.

"Anuario de Fistudios Medievales". 24 (1994)
} 
Una delle principali connotazioni che si evince dalla documentazione del periodo sui rapporti internazionali di quell'area é lo scarso rilievo che, a partire dalla metà del Trecento, ebbe il fattore religioso quale elemento di differenziazione e di contrasto. Cio si evidenzia in special modo nelle alleanze che solo in alcuni casi, diversamente che in passato, ebbero quale movente l'appartenenza ad un gruppo religioso ${ }^{2}$.

Per comprendere un simile atteggiamento da parte delle due comunità, islamica e cristiana, si deve tenere presente che nel mondo musulmano la spinta alla "guerra santa" nei confronti dei territori andalusi aveva perduto di continuità e di rilievo ${ }^{3}$. Anche in ambito cristiano, ad eccezione di alcuni episodi di lotta contro "l'infedele", si era mantenuta e sviluppata un'accentuata spinta commerciale che aveva condotto ad uno spiccato "individualismo economico". Tale impostazione, tralasciando gli ostacoli di natura religiosa, aveva teso a creare, spesso a scapito delle marinerie concorrenti, l'inserimento nei mercati islamici.

In una situazione cosl articolata, in cui le comunità cristiane e musulmane del Mediterraneo occidentale si alleavano spinte da motivazioni contingenti, non si pud parlare, se non in alcune occasioni, di realtà separate e contrapposte; al contrario furono frequenti le loro connessioni. Ed é per questo motivo (e fu una costante fino alla conquista di Ceuta) che, anche nel momento in cui si determino uno scontro armato tra gli stati dei due schieramenti religiosi, si tese a comporre in tempi rapidi la situazione bellica avendo quale principale finalità il ripristino dei rapporti commerciali ${ }^{4}$.

\footnotetext{
${ }^{2} \mathrm{~A}$ partire dalla fine del XIII secolo le alleanze non furono concluse in considerazione dell'appartenenza religiosa. Alcuni esempi: nel 1282 il dominio su Tarifa determinò una coalizione che vide uniti paesi musulmani e cristiani; nel 1356 Pedro I di Castiglia concluse una tregua con Granada nella guerra contro Pietro IV d'Aragona;(cfr. M. A. LADERO QUESADA, Grancada. Historia de un pais islamico, Madrid, 1989, pp. 138-140; 159-160)

${ }^{3}$ Dalla metà del XIII secolo si verificò un lungo periodo di pace; cfr. M. A. LADERO QUESADA, Granada, Historia de un país islanico, cit., pp. 155-165.

'Tra gli avvenimenti che hanno portato alla conquista di località nell'Africa settentrionale, il dominio su Gerba da parte di una spedizione siculo aragonese é stato uno delle più duraturi (1384-1335); Tripoli fu occupata nel 1146 per sei mesi; Mahadiya per undici anni (11481159), cosi come pure per un breve periodo Soussa, Sfax c Gabes a partire dal 1146. Del resto, affernna Thomaz, questi eventi, così come altri (esemplare dell'impossibilita di conseguire un risultato, per le numerose difficoltà che la spedizione dovette affrontare, l'incursione del 1395 a Mahadiya), non ebbero un effetto durevole perché non riuscirono a condurre una politica sistematica di conquista o ad assicurare alıneno assistenza e approvvigionamento alle posizioni acquisite; cfr. L. F. THOMAZ, Le Portugal et l'Afrique au XV siècle. Les debuts de l'expansion, Centre de estudos da Historia e Cartografia antiga, Serie separatas, 221, Lisboa 1989, p.7.
} 
In generale si pud quindi affermare che solo nel momento in cui l'intento religioso si collego e coincise con finalità di altra natura vennero a crearsi due blocchi contrapposti ciascuno dei quali si irrigidiva nella lotta in nome della propria fede.

Le stesse considerazioni si ripropongono in relazione alle azioni di corsa e di pirateria di quel periodo; gli assalti e i depredamenti in mare non furono motivati necessariamente dal fattore religioso che in ogni caso costituiva una ragione importantes; l'opportunità economica, che a volte si ammantava di idealità per perseguire finalità diverse da quelle denunciate, era comunque l'elemento fondamentale che spingeva ad intraprendere le imprese piratesche nelle quali coloro ch e effettuavano l'azione e coloro che la subivano potevano essere indifferentemente cristiani o musulmani.

La conquista portoghese di Ceuta del 1415 , per le conseguenze che ebbe, sembro aver prodotto un rinnovato schieramento. In effetti, anche se formalmente l'impresa portoghese fu salutata con grande soddisfazione dagli altri stati cristiani, e in definiva il risultato fu in quella direzione ${ }^{6}$, cio non corrispose ad una politica necessariamente ostile di questi verso gli stati magrebini.

Il sovrano portoghese João I aveva attuato nei confronti delle "nationes" cristiane che agivano nel Mediterraneo occidentale un colpo di

\footnotetext{
SIbn Khaldoun verso l'anno 1382 descrive l'organizzazione della corsa a Bugia; in maniera analoga l'attività piratesca si dovetle svolgere in altre località del Mediterraneo africano anche se si deve sottolineare che il fenomeno ebbe una diversa intensità a seconda delle località e dei periodi storici. Egli dice: "La course se fait de la manière suivante: une société plus ou moins nombreuse de corsaires s'organise; ils construisent un navire et choisissent pour le monter des hommes d'une bravoure éprouvée. Les guerriers vont faire des descentes sur les cotes et les îles habitées par les Francs; ils y arrivent à l'improviste et enlevent tout ce qui leur tombe sous la main; ils attaquent aussi les navires des infidèles, s'cn emparent très souvent, et rentrent chez eux chargés de butin et de prisionniers"; efr. L. M. MAS LATRIE, Traités de paix et de cominerce et documents divers concemant les relations des Chrétiens avec les Arabes de l'Afrique septentrionale au Moyen Age, vol. I, Paris, 1866, p. 237.

${ }^{6}$ Sulle ripercussioni che la conquista portoghese ebbe in Aragona, cfr. M. ARRIBAS PALAU, Repercussión de la conquista portuguesa de Ceuta en Aragón, "Tamuda", 3-1 (1955), p. 15. Nella lettera di Fcrnando I d'Aragona a Joāo I del Portogallo del 10 ottobre del $1415 \mathrm{si}$ legge sulla conquista di Ceuta: "Vuestra letra hauemos recebido sobre la presa feyta por vos de Cepta de manos e poder de infieles de la qual hauemos hauido muy singular e asenyalado plazer como sea cosa que toca a seruicio de dios e exaltacion de la fe catholica a que todos los fieles christianos en special Reyes e principes son obligados e augmentacion no poca de vuestro honor e fama". Zurara riferisce, nella Crónica do Conde D. Pedro de Menezes, che i veneziani che passavano nello Stretto per raggiungere le Fiandre, nel vedere che i portoghesi si erano impossessati di un naviglio musulmano"ouveraō muito prazer quando viraō como traziam aquella presa, fazendo muita cortezia aos Capitāes das Fustas, louvando muito a El Rey de Portugal pela continuaçaō da guerra, que fazia contra os infieis".
} 
mano in quanto l'impresa di Ceuta non corrispose ad un programma comune precedentemente enunciato e concordato. La meta dell'impresa infatti era stata rigorosamente tenuta segreta per l'intera durata dell'allestimento della flotta e solo poco prima della conquista fu resa pubblica? .

Il risultato che ne segul cred una nuova situazione politicoeconomica che si rivelo vincolante per i mercanti delle marinerie concorrenti che avevano dominato la scena fino a quel momento, e specialmente l'Aragona e Genova. I contatti che si vollero mantenere con il Magreb occidentale furono fortemente condizionati dagli avvenimenti portoghesi di Ceuta. Malgrado le assicurazioni di estraneita all'attacco bellico per evitare ritorsioni ${ }^{8}$, divenne inevitabile subire le reazioni negative della dinastia marocchina dei merinidi?.

L'entusiasmo con cui fu accolto il positivo esito della spedizione portoghese nascose pertanto la consapovolezza dello smacco subito sotto il profilo economico. Parallelamente alle difficolta incontrate con la dinastia merinide, si attuava un profondo cambiamento: l'impossibilità di mantenere la stessa libertà di movimento, nell'ambito del commercio e della pirateria dello Stretto, che si era avuta in precedenza. Il Portogallo aveva creato a Ceuta una costante "sentinella" che effettuava un rigido controllo sull'andamento complessivo della navigazione e dei traffici dello Stretto, sia musulmani sia cristiani.

\footnotetext{
${ }^{7}$ Gomes Eanes De ZurArA, Crónica da tomada de Ceuta, Publicaçōes Europa-América, 1992 , p. 180.

${ }^{8}$ La lettera a Abou Sa'id del Marocco da parte di Fernando I d'Aragona, del 18 novembre 1415 , lamenta le rappresaglie che si erano avute contro $i$ cristiani stabiliti in quel territorio per ritorsione a causa della conquista di Ceuta. Fernando chiarisce la sua estraneità e quella del re di Castiglia all'accaduto: "Nos ni el dito Rey de Castiella, no hauemos agreuiado ni maltractado los moros qui stan, o son presentes en los nuestros Regnos e tierras, o del dito Rey de Castiella, antes siempre los hauemos preseruado de todo sinistro, e feiyo justicia egualment a todos por que coino sea todo muyto contra toda justicia e buena razon inquietar e agreuiar los jnnocentes por los pecadores, o qui han feyto danyo a vos, o en vuestro Regno. Rogamos vos muyt affectuosament que queriendo fazer plenament justicia a los sobreditos e a todos otros christianos presentes en exos vuestros Regnos e tierras no maltractedes agrauiedes, o molestedes por la dita razon...". Altro reclamo simile porta la data del 26 novembre 1415; cfr. M. ARRIBAS PALAU, Repercussión de la conquista portuguesa de Ceuta en Aragón, cit., pp. 10; 15-19.

${ }^{9}$ Afferma Mas-Latrie che, malgrado gli sforzi fatti da una parte e dall'altra, gli schiavi musulmani erano numerosi in Europa e ancora più numerosi gli schiavi cristiani cristiani in Africa; cfr. L. M. MAS LATRIE, Traités de paix et de commerce, cit., pp. 402-404.
} 
Anche se é vero che l'attività dei corsari portoghesi a Ceuta fu da quel momento in poi indirizzata contro i musulmani, ciò derivo non da una consapevole posizione che favorisse gli stati cristiani i quali fino allora avevano operato in cuell'area, ma dal totale rovesciamento della situazione politico-economica che aveva precipuamente come unica finalita l'affermazione del Portogallo. I lusitani, divenendo arbitri degli accadimenti dello Stretto, poterono sviluppare la politica per loro più conveniente. L'intento perseguito da João I prevedeva che i vascelli delle marineric cristiane potessero navigare nello Stretto senza difficoltà di sorta in modo che l'inserimento di mercanti stranieri e l'arrivo di mercanzie provenienti dal Mediterraneo e dalle Fiandre e dall'Inghilterra contribuissero allo sviluppo del commercio delle città costiere del Portogallo.

All'epoca della spedizione di Ceuta la corsa aveva oltre ad implicazioni di natura pratica un valore ideale. João I, riferisce Matteo Pisano nella sua Cronaca sulla conquista della citta magrebina ${ }^{10}$, intendendo che gli Infanti "insignibus militaribus decorari", ritenne di poter realizzare, nel migliore dei modi, tale progetto decidendo "in Africam eos traijcere ... ad piraticam artem exercendam" "I. Non quindi tornei e feste per un anno intero, "follie e banchetti" ${ }^{2}$, come inizialmente era stato previsto per celebrare un simile evento che conferiva dignità di cavalieri a figli di re, ma assalti e depredamenti nei confronti di vascelli e territori musulmani. Per ottenere un simile decoro si richiedeva una dura esistenza "in cui fame e sete sarebbero stati uniti ai rigori del sole estivo e del freddo invernale"13. L'assalto a Ceuta e la conseguente attività corsara erano quindi in stretta relazione e facenti parte di una sola realtà. Il progetto nella sua totalità era inteso quale "serviço de Deus". Ciascuna delle due componenti era infatti caratterizzata dalla lotta contro "i nemici defla fede", unico elemento qualificante per un buon cristiano che prevedeva "più che la vita, il sacrificio della morte con gloria"14.

\footnotetext{
${ }^{10}$ Matthaeus de PISANO, De bello Septensi, in "Colleç̧aō de livros ineditos de historia portuguesa dos reinados de D. Jonō I, D. Duarte, D. Affonso V e D. Joaõ II", I, Lisboa, 1790, p. 11.

"Matthacus de PISANO, De bello Sepiensi, cit., p.ll.

${ }^{12}$ Matthaeus de PISANO, De bello Sepiensi, cit., p.11.

${ }^{13}$ Matthaeus de PISANO, De bello Septensi, cit., p. 11.

${ }^{14}$ Matthacus de PISANO, De bello Septensi, cit., p. 13.
} 
Nell'indicare alcune linee direttrici dell'affermazione marittima portoghese e della conseguente attività della pirateria e della corsa nell'estrema parte del Mediterraneo ọcidentale, negli anni che precedettero la conquista di Ceuta, si deve tenere presente che lo stato atlantico si era affacciato come potenza marittima solo a partire dagli ultimi decenni del XIV secolo con il re Fernando. Il sovrano aveva dato un forte impulso alla navigazione e aveva operato positivamente a formare quel gruppo che si puo chiamare "borghesia marittima". La spinta in tal senso si volse da un lato alla costruzione di navigli che fu promossa da relative ordinanze, dall'altro alla creazione di un organismo, la Companhia das naus che puo essere definita, il primo episodio di assicurazione marittima in Portogallo ${ }^{15}$.

Nell'ultimo scorcio del XIV secolo la marineria portoghese ebbe pertanto un notevole impulso e all'inizio del successivo aveva già compiuto notevoli progressi sia in direzione del Mediterraneo sia dell'Atlantico. Nel Mediterraneo e soprattutto in quello aragonese, negli anni che precedettero la spedizione portoghese, si ha notizia di loro azioni a danno di vassalli del sovrano Fernando $\mathrm{I}^{16}$. Segnalati nel 1412 da diverse parti del Regno, i pirati lusitani assaltavano prevalentemente le coste delle isole Baleari impadronendosi di musulmani sudditi del regno d'Aragona ${ }^{17}$.

Anche se si tratta di eventi sporadici, la loro presenza nel Mediterraneo mostra l'intraprendenza acquisita e la volontà di inserimento tra le grandi potenze marittime. "Portugal en el siglo XV parece necessitar de apoyos mediterraneos", afferma a ragione Adão de Fonseca ${ }^{18}$. La pirateria e la corsa, ma anche gli scambi mercantili ${ }^{19}$, si presentarono un

\footnotetext{
${ }^{15}$ Sulle concessioni ai mercanti di importanti privilegi, efr. H. Gama BARROS, Historia da Adıministraçđo publica em Portugal nos seculos XII-XV, Lisboa, 1950, p. 360.

${ }^{16}$ Sulla frequenza di vascelli corsari portoghesi nel Mediterraneo, efr. L. Adão de FONSECA (Navegación y corso en el Mediterraneo occidental, Los Portugueses a mediados del siglo XV, Pamplona 1978, p. 18.

${ }^{17}$ Sulla frequenza dei pirati portoghesi nei mari dell'Aragona, cfr. A. UNAU, Riflessi dell'allestimento della flotta portoghesedestinata alla conquista di Ceuta nella politica mediterranea di Fermando I d'Aragona, (in corso di stampa in "Actas del XV Congreso de la Corona de Aragón", Jaca, 1993).

${ }^{18}$ L. Adão de FONSECA, Navegación y corso en el Medilerraneo occidental, cit., p. 21.

${ }^{19} \mathrm{Da}$ lenere presente, a proposito di comınerci mediterranei portoghesi, i traffici di prodotti alimentari, quale il pane ed altri viveri, con "la terra de mouros", come si vede in una lettera del re João 1 ad un suo funzionario, del 24 marzo del 1414, nella quale tali traffici vengono proibiti. Le pene previste per i trasgressori sono gravi ("sob pena dos corpos e dos averes").
} 
modo per raggiungere questo risultato e lo Stretto di Gibilterra l'ambito più idoneo dove poter agire.

Il breve braccio di mare che separa e, parallelamente nei secoli, aveva unito le popolazioni della regione iberica e di quella magrebina era assurto a particolare importanza a partire dalla fine del XIII secolo. Da quei decenni fino alla metà del secolo successivo, nel periodo indicato come "la battaglia dello Stretto" 20 , si erano verificate in quell'area situazioni altalenanti che aveva no visto con minore o maggiore incisivita l'affermarsi del potere merinide, castigliano, aragonese, granadino e genovese. Il periodo si concluse con il forte declino degli stati musulmani $^{21}$ che coincise con il rafforzamento di quelli cristiani e con l'affermazione di un loro ruolo incontrastato.

Il presupposto principale "della battaglia dello Stretto" si ravvisa nell'importanza assunta dalle rotte atlantiche sia verso Nord sia verso Sud che portarono, in un ambito geografico diverso, alla prosecuzione del fiorente commercio inaugurato due secoli prima dalle città italiane nel Mediterraneo. La nuova realtà atlantica che si stava delineando, cioé la ricerca di nuovi mercati e del modo di raggiungere per altra via quelli già noti, era strettamente collegata alla situazione mediterranea.

Le galee genovesi e veneziane, come é noto, per prime dettero l'avvio, attraversando lo Stretto, al collegamento marittimo con l'Inghilterra e le Fiandre nei medesimi anni in cui i genovesi Vadino e Ugolino Vivaldi si volgevano lungo la costa dell'Africa occidentale (1291) e i mercanti genovesi commerciavano con ottimi profitti a Salé, Massa e Arzila.

In quegli stessi decenni lo Stretto di Gibilterra mantenne anche la funzione di passaggio per giungere dal Magreb nel sud della penisola iberica. I merinidi non avevano rinunciato alle terre dell'Andalusia anche perché il djiad conferiva loro il prestigio necessario di cui avevano bisogno per il controllo della situazione interna del proprio territorio. Dopo aver

Il sovrano sanciva che la inclà dei beni confiscati andassero a coloro che denunciavano i colpevoli; Cfr. Monumenta Henticina, vol. II, Coimbra 1960, pp.75-76.

${ }^{20} \mathrm{M}$. A. LADERO QUESADA, Granada. Historia de un pais islámico (1232-1271), cil., p. 134-156; v. dello stesso autore, Castilla, Gibraltar y Barberia (1252-1516), in "Actas del Congreso Intemacional "El Estrecho de Gibraltar", Ceuta 1987, II, Madrid 1988.

${ }^{21}$ Sulle motivazioni che portarono al declino degli stati musulmani, efr.I. HRBFK, La désiniégration de l'unité politique du Maghreb, in "Histoire Générale de l'Afrique", IV, L'Afrique du XIle au XVIc siecle, Unesco/NEA, pp. 120-121. 
preso Ceuta nel $1273^{22}$, il sultano Abou Youssef Yacoub in soli dieci anni (1275-1285) aveva passato lo Stretto cinque volte nel tentativo di riprendere in Andalusia il controllo di una situazione divenuta particolarmente complessa a causa di repentini mutamenti di alleanze ${ }^{23}$.

Un evento che illustra l'importanza che avrebbe assunto lo Stretto e il blocco navale che l'Infante di Castiglia, Sancho, riuscl ad attuare per opporsi a Abou Youssef e che determino il suo ripiegamento in Africa ${ }^{24}$. Tale avvenimento ha in sé un notevle rilievo perché mostro come il controllo navale di quel mare determinasse un'azione deterrente sui governi dei territori che in esso si affacciavano. Il risultato positivo conseguito dalla Castiglia mediante la creazione di una barriera navale tra l'Andalusia e il Magreb che ostacolava gli aiuti e il rientro delle truppe sulle coste magrebine, costitul un'importante presupposto per le nazioni cristiane, quale il Portogallo, the in seguito baseranno la loro potenza sul dominio dello Stretto, sulla forza navale, sulla consistenza delle flotte e del loro armamento.

Il blocco dello Stretto attuato dall'infante Sancho dette l'avvio anche se lentamente al predominio navale delle nazioni cristiane nel Mediterraneo occidentale che derivo soprattutto dalla diminuita importanza dei merinidi in quel settore. L'evento corrispondeva alla volontà di espansione territoriale e di inserimento commerciale in Africa della Castiglia che di ll a pochi anni si sarebbero concretizzati nel Trattato di Monteagudo stipulato con l'Aragona nel 1291, e peraltro rimasto inattuato, mediante il quale venivano individuate nel Magreh le due aree di influenza ${ }^{25}$.

\footnotetext{
${ }^{22}$ Sulle difficoltà incontrate dal sultano inerinide nella soltomissione di Ceuta, cfr. $\mathrm{H}$. FERHAT, Sabta des origines au XIV e siècle, Rabat, 1993, pp. 244-246.

${ }^{23}$ Il re di Castiglia Alfonso $X$ si cra recato in Gernania per cercare di ottencre la corona imperiale; in gran parte la tensione del re castigliano era rivolta a raggiungere tale ohicttivo. In Andalusia il sovrano, nel tentativo di controllare Muhamınad II e di impedire che il suo regno si fortificasse, riusci in un primo momento ad avere dalla sua parte gli emiri di Malaga e di Guadix, ma questa alleanza non ehhe una lunga durata per l'intervento granadino che riusci a riportare i ribelli alla sollomissione; clir. B. LUGAN, Histoire du Maroc. Des origines à nos jours, Paris, 1992, p. 111 .

${ }^{24}$ M.A. LADERo QUJiSADA, Granada. Historia de un pais islámico (1232-1271)cit., p. 138

${ }^{25}$ Il patto fu stipulato tra Jaine II d'Aragona e Sancho IV di Castiglia e il limite di influenza tra $\mathrm{i}$ due stati fu fissato al fiume Muluya, in modo che, se si fosse attuata la suddivisione, il Maroceo sarehbe spellato alla Castiglia, Algeri, Tunisi e le terre verso Levante sarebbero anadate alla Catalogna; cfr. F. SOI.DI:VILA, Sintesis de historia de Cataluña, Barcelona, 1973, p. 120; P. PRIETO Y LloVIiRA, Politica aragonesa en Africa hasta la inuerte de Fertando el Católico, Madrid 1952, p. 54.
} 
Nei decenni centrali del XIV secolo perdeva dunque di vigore "la battaglia dello Stretto" per le gravi difficolta che i sultani merinidi dovettero affrontare all'interno del proprio territorio e che ebbero una forte risonanza nel Mediterraneo occidentale.

La morte per assassinio del sultano Abou Inan aveva inaugurato un periodo di gravi crisi dinastiche che ebbero la loro espressione più esplicita nel numero dei sultani che, in un tempo breve, presero il potere: in appena sedici anni, dal 1358 al 1374, si succedettero diciassette sultani merinidi e di questi, sette perirono assassinati mentre cinque furono depost ${ }^{26}$. Una ragione della loro debolezza risiedeva nel potere dei vizir (strumenti di vere e proprie consorterie feudali che si trovavano nel Sud del paese, nelle regioni orientali e nel $\mathrm{Rif}^{27}$ ) che giunsero a scegliere $\mathrm{i}$ sultani e ad eliminarli quando non fossero stati di loro gradimento. La presenza di una feudalità dominante, nella seconda metà del XIV secolo e ancora all'inizio di quello successivo, aveva portato all'autonomia di vasti territori dal controllo centrale a costituira una delle ragioni della caduta della dinastia merinide.

Più stabile ed espressivo di una politica autoritaria e centralizzata fu il regno del sultano Abou Faris Abd el Aziz (1366-1372) il quale rafforzo il suo potere diminuendo l'influenza dei suoi ministri, pacifico la regione dell'Atlante, riprese Marrakech e conquisto Tlemcen e il Magreb centrale, mentre nei confronti dell'Andalusia poté inviare rinforzi ad Algeçiras e mantenere la piazzaforte di Gibilterra. Il suo regno sarà pero di breve durata $^{28}$. I suoi successori dal 1374 al 1393 legheranno le vicende del sultanato marocchino a quelle di Granada, il cui emiro Muhammad V (1354-1391) svolse in effetti in alcuni periodi una vera tutela sul loro territorio. La sua politica prevalentemente filo castigliana, di appoggio a Tlemcen e parallelamente di indebolimento dei merinidi era indirizzata a

\footnotetext{
${ }^{26} \mathrm{Cfr}$. B. LugAN, Histoire du Maroc. Des origines a nos jours, cit., p. 119.

27J. Brignon, A. AMine, B. Boutaleb, G. Martinet, B. Rosemberger, histoire du Maghreb, Casablanca, 1986, p. 167.

28J. M. ABUN-NASR, The History of Maghrib, Cambridge, 1971 (752), pp. 132-133.
} 
prevenire un loro attacco. Riuscl in tal modo, seppure per breve tempo ${ }^{29}$ negli anni 1384-1386, ad avere sotto il suo potere Ceuta e ottenne il controllo di Gibilterra.

Tra i sultani merinidi dopo Abou Abbas che ristabill l'autorità merinide a Tlemcen e ad Algeri, Abou Said Utman III (1398-1420) fu al potere durante la conquista di Ceuta. Malgrado si sforzasse di riprendere nelle sue mani il potere si scontro con i principi merinidi che a capo delle citta portavano lentamente lo stato alla disgregazione. In quello stesso periodo gli hafsidi di Tunisi poterono riprendere Algeri, Tlemcen e il Magreb centrale, mentre nella regione del Rif si ebbe una grave rivolta (1411-1412). A pochi anni dalla conquista di Ceuta quindi i merinidi versavano in una situazione di particolare difficolt ${ }^{30}$.

La crisi della dinastia marocchina si deve ricollegare anche a situazioni di estrema gravità già presenti al momento dell'affermazione merinide, che si acuirono particolarmente nei primi decenni del secolo. In particolare furono due le ragioni che contribuirono alla decadenza: il non poter disporre di una base etnica numericamente sufficiente che garantisse l'indipendenza dalle altre etnie (e, quale conseguenza di ciò, la duplice politica nei confronti delle tribù beduine, di iniziale favore e di contrasto nel momento in cui divenivano autonome) e infine la carenza di forza politica e di carisma che i merinidi ebbero in quanto non furono dei riformatori religio$\mathrm{si}^{31}$.

Grazie al potere espresso sull'intero Magreb la loro dinastia era riuscita nella prima metà del XIV secolo a dare una grande importanza all'asse carovaniero occidentale con andamento sud-nord, e a controllare le linee commerciali dell'est. Una volta frammentato il territorio dal punto di vista politico, le correnti commerciali si volsero maggiormente verso nordest.

Dopo secoli di dominio delle popolazioni musulmane di entrambe le sponde, nello Stretto di Gibilterra si venne dunque a creare un predominio

\footnotetext{
${ }^{29}$ Coloro que riuscirono a beneficiare della situazione che si era venuta a creare furono $i$ Maqil ostili a Tlemcen sui quali il sultano granadino si era appoggiato; cfr. J. BRIGNON, A. AMine, B. BoutAl.EB, G. Martinet, B. Rosemberger, Histoire du Maghreb, cit. p. 170.

${ }^{30}$ J. Brignon, A. Amine, B. Boutaleb, G. MARTinet, B. Rosemberger, Histoire du Maghreb, cit., pp. 167-168. Su Ceuta nel sec XIV, cfr. H. FERHAT, Sabta des origines au XIV e siècle, cit., pp. 250-259.

${ }^{31}$ B. Lugan, Histoire du Maroc. Des origines à nos jours, cit., p. 121.
} 
navale cristiano. La flotta merinide era numericamente modesta e non in grado di risolvere neppure i contrasti interni. Il sultano marocchino, nel 1413, per far fronte alla difficile situazione creatasi con il parente Sa'id, dovette ricorrere all'aiuto navale dell'Aragona, il cui re Fernando I attendeva l'occasione per poter ulteriormente consolidare il già fiorente commercio aragonese in quel territorio. Il sovrano si mostro disponibile all'invio di galee ma in cambio richiedeva la piazzaforte di Gibilterra che era gia appartenuta a suo nonno Alfonso $\mathrm{XI}^{32}$. Il progetto mostra quanto fosse ritenuto importante il controllo sullo Stretto. L'Aragona avrebbe ottenuto, oltre ad esiti commerciali, che si eliminassero i numerosi atti di pirateria contro i navigli aragonesi che transitavano in quel mare.

Seppure il disegno relativo a Gibilterra sia abortito in quanto la piazzaforte apparteneva al sultano granadino e non fu possibile ottenerla, la pace stabilita dall'Aragona con entrambe le nazioni musulmane, Granada e Marocco, giuocava nella direzione del controllo dell'area. Per mantenere un rapporto pacifico, il re aragonese rivolgendosi agli ufficiali del Regno prevedeva gravi pene pecuniarie per coloro che avessero arrecato danno ai sudditi delle due dinastie $e^{33}$.

Se non si puo negare che atti di pirateria da parte musulmana potessero essere attuati ai danni dei sudditi dell'Aragona nello Stretto e nella zona atlantica, anche per l'impossibilità di controllo sulla navigazione costiera da parte del potere merinide, é certo che nella documentazione aragonese di poco precedente alla conquista di Ceuta la questione degli schiavi aragonesi emerge solo marginalmente ${ }^{34}$. Nell'elencare, ad esempio, i principali argomenti che i messaggeri di Fernando avrebbero dovuto esporre al sultano Abou Said Utman III, il riscatto degli schiavi era relegato all'ultimo punto ${ }^{35}$.

Al contrario il pericolo che il "baile" di Valenza, Joan Mercader, indicava con particolare insistenza per i navigli aragonesi che giungevano

\footnotetext{
${ }^{32} \mathrm{M}$. ARRIBAS PALAU, Intercambio de eimbajadas entre Abu Sa id Utman III de Marruecos y Fermando de Aragón, in "Centro de estudios marroquíes", Tetuán 1956, pp. 37 38.

${ }^{33} \mathrm{M}$. ARRIBAS PALAU, Las treguas entre Castilla y Granada firmadas por Femando I de Antequera, Tetuán, 1956, pp. 58-59. 38.

${ }^{3}$ M. ARRIBas PalaU, Intercambio de embajadas entre Abu Sa 'id Utman III, cit., pp. 3737-38.

${ }^{35}$ M. ARRIBAS PALAU, Intercambio de embajadas entre Abu Sa 'id Utman III, cit., pp.
} 
dallo Stretto proveniva da pirati cristiani. Una ricca documentazione relativa alle missive inviate dal funzionario regio al sovrano d'Aragona, nei due anni precedenti alla conquista di Ceuta, registra esclusivamente notizie di assalti e depredamenti da parte di navigli cristiani.

Segnalazioni di mercanti genovesi di Tarifa e di Siviglia fanno intravedere la grave situazione esistente nello Stretto. Si tratta di lettere il cui contenuto riferito al sovrano dal "baile" di Valenza riguardava la navigazione dei vascelli aragonesi carichi di oro provenienti da quella regione. Le notizie riportate nelle missive del 14 e del 18 febbraio del 1414, vennero riferite al sovrano il 6 marzo dello stesso anno ${ }^{36}$.

La ragione di informazioni di tal genere da parte di genovesi non è del tutto chiara: l'anno precedente, nel 1413 , era stata firmata la pace tra l'Aragona e Genova ma cid non ci sembra motivi l'interessamento nei confronti dei pericoli che gli aragonesi avrebbero avuto nella navigazione dello Stretto. Si deve tenere presente che la situazione dei mercanti genovesi in quel braccio di mare era particolarmente florida per il loro inserimento in entrambe le aree, magrebina e granadina, e che, come ricorda Joan Mercader nel settembre del 1413, anche i vascelli aragonesi "cascun jorn van de questa çiutat (Valenza) en Granada e en Barberia" ${ }^{37}$.

E' possibile avanzare l'ipotesi che l'interessamento fosse in qualche modo collegato ai preparativi della spedizione di João I e alla condizione di timore e di pericolo in cui si trovarono i mercanti di Genova per la voce che circolava a Lisbona circa la meta sivigliana dell'impresa portoghese ${ }^{38}$. L'allarme che proveniva da un possibile attacco a Siviglia li accomunava ai vassalli dell'Aragona anch'essi in allerta e pronti a respingere la spedizione portoghese ${ }^{39}$.

\footnotetext{
${ }^{36}$ M. TINTÓ SALA, Cartas del baile general de Valencia Joan Mercader, al Rey Fermando de Antequera, Valencia, 1979, p. 192.

${ }^{37} \mathrm{M}$. TINTO SAIA, Cartas del baile general, cit., p. 176.

${ }^{38}$ Gomes Eancs de ZURARA, Crónica da tomada de Ceuta, cit., p. 122 ("algunos genoveses, estantes na cidade de Lisboa, escreveram a outros seus parceiros estantes em Sevilha, recontandolhe todo o ardimento que se trazia o reino de Portugal acerca do aviamento daquela frota. E posto que se algumas cousas dissessem de desvairadas maneiras, os mais dos sesudos criam que tuto se fazia para irem sobre a cidade de Sevilha").

${ }^{39}$ Non e di questo avviso Ricard (R. RICARD, Études sur l'histoire des Portugais au Maroc, Coimbra, 1955, p. 11, nota 1) il quale osserva, sulla scorta di quanto affermato dagli storici, Antonio Sergio e Jaime Cortesấ, che i genovesi di Lisbona poterono trovarsi d'accordo a portare avanti un'impresa che doveva avere come risultato la riduzione della pirateria barbaresca. Aggiunge però anche che l'occupazione portoghese di Ceuta avrebbe
} 
Le informazioni che i mercanti genovesi dettero al "baile" valenzano riguardavano $i$ vascelli di pirati che si trovavano in quel momento nello Stretto: si trattava di sei navigli, di cui quattro balleners e due galeotte usciti dal porto di Siviglia; i balleners appartenevano ad un biscaglino, al conte di Niebla, ad un armatore di Cartagena e ad uno di Maiorca; le galeotte erano di Cartagena e di Alicante. Erano quindi per la lo più castigliani ma c'erano anche vascelli di provenienza atlantica e di sudditi aragonesi.

Risulta interessante constatare che il funzionario di Valenza attribuisse a questi vascelli il principale motivo di pericolo per la navigazione dei navigli aragonesi nello Stretto in quanto la loro presenza minacciosa limitava la liberta di movimento e quindi l'ingresso di oro nelle casse della Corona aragonese ("Ffan molt de dan, entre.ls altres a molts de aquest regne e a la vostra seca car totes les dobles qui devien ésser cobrades dels avers que són estats navegats en Barberia són en gran part desviades..." $)^{40}$. Le azioni di pirateria che tali navigli avrebbero potuto compiere contro navigli aragonesi erano considerate quindi un grave danno e un forte deterrente per la navigazione di quell'area; l'importazione di oro, sosteneva Joan Mercader, se si fosse ovviato ad un simile inconveniente, sarebbe potuta essere di gran lunga superiore. Per risolvere positivamente tale difficile situazione il funzionario valenzano spronava il sovrano affinché intervenisse in modo che "vostres vassalls puxen navegar segurament" ${ }^{41}$. Si doveva agire , egli affermava, nei confronti specialmente di castigliani dato che "les altres nacions prou los guarden honor per vostra reverència e temor" 42 .

potuto sminuire il ruolo di internediari che i mercanti genovesi vi svolgevano e avere come conseguenza la perdita dell'inportanza mercantile della città magrebina, cosa questa che avrebbe danneggiato il loro comınercio. Ciò in effetti avvenne, avverte Ricard, dato che nei testi a partire dal 1415 i mercanti genovesi erano assenti (Idem, p. 119). Sugli interessi genovesi nella spedizione di Ceuta, L. Adão de FONSECA (Navegación y corso en el Mediterraneo occidental, Los Portugueses a inediados del siglo XV, cit., p. 25) afferma che "en realidad, nos encontrainos ante un panoraina poco conocido, sobre el qual nos movemos con cierta dificultad". Sull'allanne che si era creato a Siviglia, Zurara dice che il vescovo della città, per la paura di un attacco, dispose la difesa; cfr. Gomes Eanes de ZuRARA, Crónica da tomada de Ceuta, cit., p. 123 ("que a cidade de Sevilha seja logo avisada e os muros reparados e os annazéns providos, e que as portas sejam bem fechadas, e as chaves entregues a homens fiéis...".

${ }^{40}$ M. TINTO SAIA, Cartas del baile general, cit., p. 192

${ }^{41} M$. TiNTÓ SALA, Cartas del baile general, cit., p. 192

${ }^{42}$ M. TINTÓ SALA, Cartas del baile general, cit., p. 192 
Pirati castigliani intercettavano ogni genere di beni e tra questi anche prodotti quali fichi e uve passe. Nel periodo della vendemmia veniva cosl annullato il guadagno che si sarebbe potuto ricavare dai beni della terra. La frutta era un prodotto deperibile, ricorda il funzionario, "que d'açi a sis meses serà tot podrit"43. Si sarebbe in tal modo perduta la somma di più di 50.000 doppie a causa delle naus e dei baleners castigliani che "se prenen totes les fustes e béns que poden atényer de vostres vassalls". I mercanti aragonesi, sosteneva ancora il funzionario regio, si sentivano "segurs de totes les nacions del món" mentre erano "guerrejats per sols castellans"44.

Anche i giurati di Valenza lamentavano nel marzo del 1414 i gravi episodi di cui erano autori i pirati castigliani il cui numero, a loro avviso, andava sempre più aumentando. La denuncia esprimeva la gravità degli atti di costoro per le conseguenze che ne sarebbero derivate al commercio aragonese: l'azione compiuta "es cosa inhumana e si axf sa a fer mes val cessar toda mercadoria..." 45 .

Questa affermazione, anche se non esclude che si verificassero parallelamente episodi di pirateria musulmana, sottolinea l'aggressività dei pirati cristiani nel Mediterraneo occidentale e induce a pensare, per la presumibile difficoltà di coesistenza di pirati musulmani e cristiani nel medesimo ambito, che le azioni dei primi potessero essere limitate dalla presenza dei secondi.

Se la documentazione aragonese pud essere ritenuta parziale e non esaustiva della situazione che si ebbe negli anni precedenti alla conquista della città magrebina, altro genere di fonti conforta l'idea di un ridimensionamento della pirateria musulmana nello Stretto. Nella Cronaca del veneziano Antonio Morosini, ad esempio, sono indicati i viaggi compiuti dalle galee della Serenissima nel Mediterraneo e nell'Atlantico. Per gli anni che precedettero la spedizione di Ceuta, e che videro il suo allestimento (1409$1415)^{46}$, non risulta alcun episodio di pirateria musulmana che avesse provocato incidenti ai navigli di Venezia che attraversavano lo Stretto. Morosi-

\footnotetext{
${ }^{43}$ M. TintO SALA, Cartas del baile general, cit., p. 232. La missiva é del 12 settembre del 1414.

${ }^{4} M$. Tinto SAla, Cartas del baile general, eit., p. 232.

${ }^{45}$ Archivo de la Corona de Aragón, Cartas reales, Femando I, n. 1310.

${ }^{46}$ A. Morosini, Chronique, Introduction et commentaire par G. Lefevre-Pontalis, Texte établi et traduit par L. Dorez, IV, Paris, 1898, pp. 244-319.
} 
ni registra il mancato arrivo nella città lagunare, il 20 gennaio del $1410^{47}$, di un vascello che viaggiava in conserva con altri tre provenienti dalle Fiandre, in quanto affondato per una tempesta all'entrata di Faro; in quel frangente, sottolinea il veneziano, erano andati perduti 30 sacchi di lana. La considerazione del naufragio e l'indicazione del carico mostra l'attenzione che il cronista poneva nel registrare gli incidenti che potevano verificarsi durante i viaggi dei navigli veneziani.

Un'ulteriore indicazione in tal senso si evince dal racconto di Zurara, nella Crónica da tomada de Ceuta, del viaggio in Sicilia che i due messaggeri di João I fecero con sosta a Ceuta, sia all'andata sia al ritorno, alcuni mesi prima della partenza della spedizione. E' indicata dal cronista la possibilità che ebbero i messaggeri di osservare indisturbati le caratteristiche del luogo e di muoversi nelle varie parti della città senza destare alcun sospetto, atteggiamento che depone in favore del pacifico inserimento di stranieri nella città e di un clima di distensione. Anche in questo caso, nessun accenno viene fatto alla situazione di pericolo per la presenza di corsari saraceni nello Stretto.

Ancora la Crónica da tomada de Ceuta offre spunti di riflessione a proposito delle notizie che circolavano in varie località e nella stessa Lisbona sulla meta segreta della spedizione. Il cronista portoghese chiarisce che non vi era "algum que podesse certamente ... falar na citade de Ceu$\mathrm{ta}^{48}$. Tale indicazione fa opinare che la meta dello Stretto non dovesse essere, comunemente, ritenuta importante e quindi che le difficoltà derivate dalla corsa musulmana non fossero tali da rendere possibile un intervento in quella direzione. Bisogna aggiungere che il segreto sugli intenti dell'impresa poté forse essere mantenuto proprio per l'improbabilità della meta stabilita.

Le medesime considerazioni si evincono da un'interessante ed ampia missiva al re aragonese Fernando I, dei primi mesi del 1415 , il cui autore, l'ambasciatore-spia, Rui Diaz de Vega, aveva avuto l'incarico di comunicare, da Lisbona, al sovrano le voci e quanto si riferisse alla spedizione e alle sue finalità. Non solo nella città portoghese non circolava alcuna notizia sulla meta marocchina (egli indica ben dodici possibilità), ma una simile eventualità non era avvertita neppure nell'ambiente mercantile

\footnotetext{
${ }^{47}$ A. Morosini, Chronique, cit., IV, p 312.

${ }^{48}$ Gomes Eanes de ZURARA, Crónica da tomada de Ceuta, cit., p. 120.
} 
aragonese che, per i suoi traffici con il Magreb occidentale doveva ben conoscere la situazione in quell' area $^{49}$.

La notizia che si trattasse di Ceuta o di Gibilterra fu data al sovrano aragonese da Diaz de Vega solo il 28 luglio, pochi giorni prima della partenza della flotta quando probabilmente qualche indicazione comincio a trapelare ${ }^{s_{0}}$.

Nell'individuare le finalità dell'impresa portoghese che spinsero il sovrano a partire, Zurara ne evidenzia tre: la lotta contro gli infedeli, l'offerta della propria persona al pericolo per "serviço de Deus" e infine la possibilità per gli Infanti di divenire cavalieri dopo aver superato prove gravose. La guerra contro i nemici della fede era indicata come l'unica esperienza bellica che potesse essere attuata dai cristiani: si propugnava la "guerra contra quaiquer infiéis, assim mouros como gentios, ou quaiquer outros que, por algum modo, negarem alguns dos artigos da Santa Fé Católica"s!.

Se queste ragioni giustificavano la volontà di andare contro i musulmani, resta non precisata nella Crónica, la motivazione che doveva portare la spedizione contro Ceuta. Il cronista sottolinea che la presa della città sarebbe stata "o primero começo da destruição dos moros", e ancora il primo dominio in Africa da parte di un "Rei de Espanha"s2; nessun riferimento viene fatto alle ragioni per le quali era stata scelta proprio quella città musulmana. Un analogo risultato si sarebbe potuto ottenere con la presa di un'altra citta magrebina che al pari di Ceuta potesse essere definita "grande e nobre e asada para se tomar".

Zurara, inoltre, evidenziando i fattori di dubbio che si opponevano alla realizzazione del programma di conquista e trovando per ciascuno di essi una soluzione positiva che avrebbe dovuto confortare João I nella sua decisione, non dà risposta ${ }^{53}$ all'unico quesito che precipuamente si riferiva a Ceuta (e che avrebbe indicato la sua situazione nello Stretto e le finalità che si volevano ottenere), e cioé la ragione per la quale si dovesse rag-

\footnotetext{
${ }^{49} \mathrm{Cfr}$. Monumenta Henicina, II, cit., pp. 142-143.

${ }^{50} \mathrm{Cfr}$. Monumenta Henricina, II, cit., pp. 167.

"si Gomes Eanes de ZURARA, Crónica da tomada de Ceula, cit., p. 67.

${ }^{52}$ Gomes Eanes de ZURARA, Crónica da tomada de Ceuta, cit., p. 86.

${ }^{53} \mathrm{Cfr}$. G. Eanes de ZURARA, Crónica da tomada de Ceuta, cit ., pp. 72-74
} 
giungere una città "tão alongada de nós" ${ }^{34}$. Il cronista non ha quindi in nessun caso espresso una reale motivazione che spieghi la decisione della Corona portoghese. Gli stereotipi, di carattere ideale cui egli ricorre, sulla guerra cristiana, malgrado l'elemento religioso si debba ritenere importante nella concezione dell'impresa, non presentano elementi di connessione con la situazione contingente che necessariamente dovettero avere un loro importante peso.

Più aderente allo svolgimento degli avvenimenti che si verificarono nello Stretto appare l'atteggiamento di Zurara nella Crónica do Conde D. Pedro de Menezes nella quale sono descritte con dovizia di particolari le azioni volute dal primo governatore portoghese, Pedro de Menezes ${ }^{55}$, all'indomani della presa di possesso di Ceuta. Diversamente dalla cronaca dello stesso Zurara sugli antefatti della spedizione, in questa opera sono posti in luce con aderenza alla realtà gli eventi la cui analisi fornisce una possibile chiave di lettura degli obiettivi che l'impresa si era proposta.

Anche in questo caso si fa riferimento alla lotta ideale contro gli infedeli, "corsarios e ladroes" musulmani; ma a queste considerazioni che sembrano quasi d'obbligo si contrappone la narrazione dei numerosi avvenimenti che testimoniano una ridotta corsa saracena.

La Crónica proprio per l'abbondanza di dati sulle imprese portoghesi che si mossero da Ceuta deve essere giudicata fondamentale per stabilire quale fosse la frequenza degli episodi della pirateria musulmana in quel mare. Si deve comunque tenere presente, nel rilevare la scarsa attività della pirateria saracena, che l'insediamento portoghese servl certamente da freno per tale attività. .

Il proposito del sovrano João I appare dal racconto di Zurara, piuttosto che la lotta contro la corsa saracena, l'intercettazione e la cattura dei navigli mercantili musulmani. Utilizzando l'espressione del cronista portoghese che ben esprime le finalità perseguite negli anni successivi alla conquista, i navigli portoghesi di stanza a Ceuta ebbero quale compito precipuo quello di "sojugar aquella parte do maar, que era visinha"s6. Controllare l'area dello Stretto avendone il dominio significo concretamente

\footnotetext{
${ }^{54}$ Cfr. G. Eanes de Zurara, Crónica da tomada de Ceuta, cit., p. 68.

${ }^{35}$ Pedro de Menezes mori all'età di sessanta anni a Ceuta, il 26 settembre 1437; cfr. F. Sureda Blanes, Abyla Herculana, Santiago de Chile, Madrid, Buenos Aires 1925, p. 186.

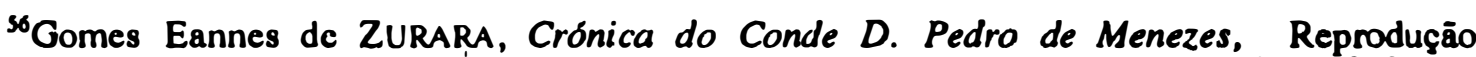
facsimilada com nota de apresentaçño por J.A. De Frcitas Carvalho, Porto, 1988, p. 310.
} 
nella vita quotidiana di coloro che si erano insediati a Ceuta intercettare tutti i navigli musulmani, carichi di mercanzie e di diverse dimensioni che transitavano nello Stretto. Le azioni contro la pirateria musulmana furono a confronto ben poca cosa.

L'ambito delle azioni portoghesi si rivelò più ampio del solo Stretto per il desiderio del Conte D. Pedro e degli hidalgos di ottenere importanti risultati che potessero compensare le gravi difficoltà che si vennero a creare a Ceuta ${ }^{57}$. Le imprese furono quindi organizzate in modo che si conseguissero in ogni caso cospicui bottini e tale compito é costantemente indicato nella Crónica come un precipuo obiettivo dei vascelli che, è sottolineato, non dovevano rimanere inattivi. I dati sulle località che potevano essere raggiunte mostrano come il desiderio di depredamento portasse $i$ vascelli portoghesi ad operare in acque anche lontane; ad esempio, in occasione di un'incursione nella zona di Malaga da parte di cinque vascelli che viaggiavano in conserva, era stato stabilito dal Conte D. Pedro che, se non fosse stato catturato alcun naviglio, "correi de longo pela costa, até o cabo de Gata, e da hi tomai a volta da Berberia, ou da huma parte, ou da outra será necessario achardes alguma presa" ${ }^{58}$.

Le località che si dovevano raggiungere variavano quindi e potevano riguardare in una medesima impresa entrambe le coste, iberica e magrebina, e località più o meno vicine a Ceuta ${ }^{59}$. Si trattò prevalentemente della zona di Malaga ${ }^{60}$ e Gibilterra $^{61}$, ma anche di Tangeri ${ }^{62}$, Alcacer Seguer ${ }^{63}$, Sale ${ }^{64}$, Larache ${ }^{65}$, tutti nodi importanti per il passaggio di

\footnotetext{
${ }^{57}$ Dice Zurara (Crónica do Conde D. Pedro de Menezes, cit., p. 274) che "os Mouros cın quanto viveraõ acerca da Cidade ...cada dia pelêjavaın, e faziaın suas escaraınuças...". Abou Sa 'id Uiman III aumentò il proprio contingente $e$ in due occasioni la citta fu sul punto di cederc; cfr. F. SUREDA BLANES, Abyla Herculama, cit., p. 186

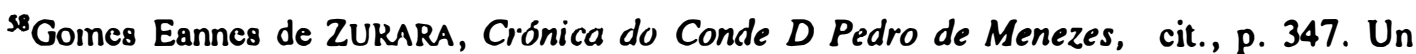
episodio analogo, idein, p. 360.

${ }^{59}$ Gomes Eannes de ZURARA, Crónica do Conde D Pedro de Menezes, cit., p. 360. 349.

${ }^{60}$ Gomes Eannes de Zurara, Crónica do Conde D Pedro de Menezes, cit., pp. 347, 348,

${ }^{61}$ Gomes Eannes de ZurarA, Crónica do Conde D Pedro de Meneses, cit. pp. 315; 348.

${ }^{62}$ Gomes Eannes de ZURARA, Crónica do Conde D Pedro de Menezes, cit. pp. 349; 385.

${ }^{63}$ Gomes Eannes de ZURARA, Crónica do Conde D Pedro de Menezes, cit., p. 410. 407.

${ }^{64}$ Gomes Eannes de ZURARA, Crónica do Conde D Pedro de Menezes, cit. pp. 384; 405;
} 
vascelli carichi di mercanzie. La vendita delle merci depredate poteva anche avvenire in citta lontane della costa magrebina, quale Tunis ${ }^{56}$.

Gli assalti a navigli musulmani che trasportavano mercanzie e il loro depredamento risultano molto frequenti ${ }^{67}$ tanto che "casy cada semana avia presa grande, ou pequena" ${ }^{68}$. Se giungevano segnalazioni sul passaggio di vascelli mercantili ${ }^{69}$, si provvedeva all'armamento di uno o più navigli (si ha notizia anche di partecipanti non portoghesi ${ }^{70}$ ) i quali si appostavano per cogliere di sorpresa il naviglio nemico ${ }^{71}$. A volte si effettuavano appostamenti per osservare indisturbati le operazioni di carico ("bem he, que viam hir os barcos pera ella com alguma fardagem, que os mercadores queriam levar pera sua viagem ${ }^{72}$ ").

L'attesa nella speranza di avvistare un naviglio carico di merci da attaccare si poteva prolungare a lungo, come nel caso dei due brigantini che "jouvessem de mar em roda toda a noite" prima di poter procedere all'assalto di due zavras cariche di frumento e di farina ${ }^{73}$, o della galeotta di Cartagena e di un brigantino che rimasero presso le isole di Alfahiba per tre giorni "aguardando se atravessariam Navios dos contrairos" senza conseguire alcun risultato ${ }^{74}$.

\footnotetext{
${ }^{65}$ Larache venne incendiata dai portoghesi; cfr. Gomes Eannes de ZURARA, Crónica do Conde D Pedro de Menezes, cit., pp. 383; 405.

${ }^{66}$ Gomes Eannes de ZURARA, Crónica do Conde D Pedro de Menezes, cit., p. 362

${ }^{67} \mathrm{Cfr}$. sui navigli musulmani carichi di mercanzie che furono catturati negli anni appena successivi alla conquista di Ceuta, Gomes Eunnes de ZURARA, Crónica do Conde $D$ Pedro de Menezes, cit., pp. 310; 312-315; 346; 348; 349; 361; 362; 368; 369; 385; 388; 390; 399; 405; 410; 416; 418.

${ }^{68}$ Gomes Eannes de Z1'RARA, Crińnica do Conde D Pedro de Menezes, cit., p. 388.

${ }^{69} \mathrm{Le}$ segnalajioni avvenivano anche per iscritto da località costiere che avvertivano del passaggio di vascelli musulmuni; da Tarifa, ad esempio, era stato comunicato che "hum lenho d'Alcaçar era passado a Gihraltar pera llevar inessagciros, e que dous caravos estavāo carregados coin hestas c outrus cousa, que levaväo pera EI Rey de Fez de presente"; cfr. Gomes Eannes de Zurara, Ciónica dis Conde D Pedro de Menezes, cit., p. 388.

${ }^{70}$ Gomes Eannes de Zuraka, Crínica do Conde D Pedro de Menezes, cit., pp. 360; 378; $388 ; 390 ; 400,401$.

"Gomes Eannes de Zuraka, Crómica do Concle D Pedro de Menezes, cit., p. 311.

${ }^{12}$ Gomes Eannes de Zuraka, Crónica do Conde D Pedro de Menezes, cit., p. 312.; in questo caso le mercanzic the crano state caricate erano di particolare valore dato che si trattava di" especialinente seda fina, e roupa talhada, e muita moeda d'ouro, e de prata, afora fruita de que levavaí a inaior parte do latro...". del valore complessivo diu 70.000 corone.

${ }^{73}$ Gomes Eannes de Zl'rara. Crónica do Conde D Pedro de Menezes, cit., p. 410.

${ }^{74}$ Gomcs Eannes de ZURARA, Crónica do Conde D Pedro de Menezes, cit., p. 360.
} 
Oltre all'acquisizione di vascelli e di mercanzie si giungeva alla cattura di uomini. I mori si difendevano a volte strenuamente ma gli scontri ${ }^{75}$ si concludevano spesso con la morte, il ferimento e l'annegamento di molti di loro. Nel tentativo di salvare la propria vita, essi lanciavano a terra il vascello portandolo ad incagliarsi lungo la costa e dandosi alla fuga ${ }^{76}$.

Solo raramente le azioni si concludevano con gravi perdite dei portoghesi. Quando il combattimento si faceva particolarmente cruento, anche costoro, sebbene fossero forniti di un armamento adatto per l'attacco, rischiavano la vita ${ }^{7}$. Lo scontro si poteva presentare difficile in particolar modo quando il numero dei musulmani risultava superiore al previsto ${ }^{78}$. Nel caso di Gonçalo Vazques si tratto di combattere contro 77 mori gran parte dei quali si trovava precedentemente sotto coperta e che si mostrarono particolarmente agguerriti cogliendo di sorpresa l'avversario. Alvaro Fernandes Palenço, capitao di navigli, che aveva partecipato a numerose azioni contro i musulmani, era stato fatto prigioniero e in seguito riscattato con una somma di 1500 doppie $^{79}$. Ma tali eventi nella Crónica do Conde $D$. Pedro de Menezes risultano sporadici e cio si deve probabilmente attribuire alla disparità di armamento tra $i$ contendenti dovuta alla diversità di intenti.

Gli attacchi nei confronti di navigli mercantili musulmani furono dunque frequenti e generalmente redditizi anche perché erano associati a scorrerie sulla terraferma in localita di passaggio dei mercanti o in luoghi in cui si trovavano prodotti che potessero presentare un qualche interesse economico ${ }^{80}$.

Nella Crónica di Zurara di gran lunga meno incisive appaiono le azioni dei corsari musulmani. Scarsa é infatti la rilevanza che é loro attribuita. L'argomento viene affrontato da Zurara in alcuni casi a margine della descrizione di cattura dei navigli mercantili. Oltre al numero esiguo di eventi segnalati stupisce il modo sbrigativo che quasi costantemente il

\footnotetext{
${ }^{75}$ Gomes Eannes de Zurara, Crónica do Conde D Pedro de Menezes, cit., p. 416.

${ }^{76}$ Gomes Eannes de Zurara, Crónica do Conde D Pedro de Menezes, cit., p. 311.

${ }^{71}$ Gonçalo Vazques de Ferreira, pur ferito, aveva potuto salvarsi grazic all'intervento, in extremis, di un balestriere che gli aveva lanciato un pavese; cfr. Gomes Eannes de ZURARA, Crónica do Conde D Pedro de Menezes, cit., p. 415.

${ }^{78}$ Accadde a Gonçalo Vazques; cfr. Gomes Eannes de ZURARA, Crónica do Conde $D$ Pedro de Menezes, cil., p. 415.

${ }^{79}$ Gomes Eannes de Zurara, Crónica do Conde D Pedro de Menezes, cit., p. 535.

${ }^{80}$ Goines Eannes de ZURAR^, Crónica do Conde D Pedro de Menezes, cit., pp. 351-352.
} 
cronista adotta nella descrizione. A volte l'avvenuta presa dei vascelli corsari é enunciata senza dar conto di come si fossero svolti i fatti. Descrivendo l'impresa di Afonso Garcia, ad esempio, Zurara ricorda in maniera dettagliata il depredamento da lui attuato di una "barca" mercantile carica di "muitas coisas de grande valor" mentre solo a conclusione dell'esposizione su tale accadimento fa riferimento al fatto che fu proprio Afonso Garcia "o que desharatou Boboramonte hum Mouro grande cossairo, que morava em Tanger", e colui che aveva anche vinto il figlio del corsaro Esnarigado, Bemirgáo. L'unica notazione che egli introduce in tale racconto riguarda la cattura delle fustas dei corsari e il loro trasporto a Ceuta ${ }^{81}$.

Nella sola descrizione della disfatta di Esnarigado, apostrofato come "hum valente Cosairo Mouro"82, Zurara si dilunga e fornisce particolari sui diversi momenti dell'inseguimento e della cattura della sua fusta e di quelle dei corsari che agivano con lui. Non si accenna pero alla sua sorte. Si sa comunque che era ancora presente sul mare nel periodo successivo $^{83}$. L'attenzione particolare nei confronti di tale corsaro e la scarsa considerazione di altri episodi di pirateria musulmana fa ritenere che in quegli anni non se ne siano verificati altri di particolare rilievo ${ }^{84}$.

Se ne deduce che la corsa musulmana non dovette pertanto avere una particolare incidenza sulla navigazione dello Stretto negli anni successivi alla conquista prima che avesse inizio l'accerchiamento da parte musulmana ${ }^{85}$; se cosl non fosse, per il desiderio di magnificare le azioni dei

\footnotetext{
${ }^{81}$ Gomes Eannes de ZURARA, Crónica do Conde D Pedro de Menezes, cit., pp. 312-315.

${ }^{2}$ Gomes Eannes de ZuRara, Crónica do Conde D Pedro de Menezes, cit., pp. 343, 344. Esnarigado, agcndo con altri due corsari, aveva futto "escalla em terra, onde se acaba o muro d'Almina em huina calla, que he da parte do levante". In un secondo momento la fusta di Esnarigato e quelle dei corsari che avevano agito con lui vennero prese. Zurara aggiunge una nolazione su come considerasse la pirateria musulmana; nel momento in cui $i$ mori di Esnarigado videro imminente il pericolo, il corsaro avrebbe esortato i suoi con queste parole: "que estevessem quedos, e que não temessem os Christãos; caa homens eram como elles, e que leixassem chegar, e achariam quem lhes mostrasse, quanto havia de bom Mouro a bom Christão"; il cronista esalta in tal modo la perizia dei portoghesi e la evidente superiorita della base di Ceuta; ma egli sembra riferirsi anche alle difficolta che incontrava la pirateria saracena. 519.

${ }^{83}$ Gomes Eannes de ZuRARA, Crónica do Conde D Pedro de Menezes, cit., pp. 417, 516-

"Gomes Eannes de ZURARA, Crónica do Conde D Pedro de Menezes, cit., p. 346.

${ }^{85}$ Gomes Eannes de ZURARA (Crónica do Conde D Pedro de Menezes, cit., p. 494) afferma che l'accerchiamento ha avuto luogo quatto anni dopo la presa di Ceuta, nel mese di agosto.
} 
portoghesi, Zurara avrebbe dato maggior risalto alle imprese che si rivolgevano contro i corsari musulmani. Alla medesima conclusione conduce il fatto che il cronista, nel riferire l'opinione degli hidalgos che intendevano stanziarsi nella città conquistata opponendosi a coloro che ritenevano fosse meglio abbandonarla, non faccia alcun cenno ai pericoli che $i$ saraceni avrebbero potuto provocare ${ }^{86}$. Siamo quindi propensi a ritenere che la pirateria e la corsa musulmana non fossero avvertite come un problema tanto rilevante, come é stato finora affermato ${ }^{87}$ sia nel periodo precedente alla presa di Ceuta sia negli anni appena successivi.

Concludendo, sulla scorta delle riflessioni compiute, la principale caratterizzazione della situazione nello Stretto, sia negli anni che precedet-

\footnotetext{
${ }^{86}$ G. Gonzalves Busto, Aspectos del corso en el Estrecho de Gibraltar. Primer siglo de Ceuta portuguesa, "Actas del Congreso Intemacional 'El Estrecho de Gibraltar'", Ceuta, 1987, II, Madrid, 1988, p. 298. Sccondo Gonsalbcs Busto, tra le ragioni che avrebbe addotto Joāo, nella cronaca di Zurara, nessuna indica il pericolo della navigazione della corsa nello Stretto, cosa questa che induce a ritenere che tale pericolo non esisteva o che all'inizio del XV secolo cra minimo o quasi nullo.

${ }^{87}$ Il principale sostenitore dell'importanza della pirateria musulmana tra le finalità della conquista di Ceuta è Jaime CORTESAO (Os descobrimentos Portugueses, cit., II, pp. 288-289). Lo storico portoghese è dell'avviso che le azioni piratesche, all'epoca in cui Joấ I decise la spedizione nello Stretto, erano raddoppiate e che avevano determinato quello "estado de asfixia em relação aos productos do Levante, como aliás aos de todo o comércio mediterránico". Lo storico portoghese afferna che il prezzo del pepe nel periodo della conquista di Ceuta e nei decenni successivi illustra bene il mutamento avvenuto a favore del rifomimento di prodotti orientali in Portogallo. Il prezzo era passato dai 32 shillings ai 16,13 e 9 shillings. A nostro avviso, considerando la situazione della pirateria cristiana dello Stretto prima della conquista portoghese, così come eınerge dalla documentazione aragonese citata, tale diminuzione si potrebbe attribuire non solamente all'assenza della pirateria musulmana ma anche di quella cristiana. La tesi di Cortcsīo si basa in parte su quanto aveva affermato Mas- Latrie il quale sostiene che il discgno di João I inlcse colpire la pirateria musulmana. Il sovrano portoghese "s'était plaint souvent aux sultans mérinides des agressions parties de leurs provinces septentrionales. Fatigué de l'inutilité des ses représentations, il se décida a venger ses griefs et à tenter de s'établir au centre de ces populations hostiles, pour être à même de protéger les passages de ces vaisseaux de l'Océan dans la Mediterranée". Mas Latrie, affermando che "la necessité de pouvoir à la sécurité de lcurs côtes amena les Européens à agir cux mêmes contre les populations chez qui les sultans ne pouvaient plus assurer le respect des traités et de leur propre autorités", mette in luce l'incidenza dei danni provocati dai musulmani. Ma quando si sofferma sulla pirateria dei cristiani $e$ intende stabilire "quelle part de résponsabilité incomberait aux Chrétiens, durant la période qui succéda aux grandes invasions sarrasines", lo storico dice che $\mathrm{i}$ cristiani hanno avuto "une part trés-considérables dans l'ensemble des pillages et des dévastations maritimes que nous rejetons trop facilement au compte des barbares. Si les Chréticns nous paraissent avoir plus souffert de la piraterie, c'est qu'ils avaient un commerce plus considérable, des côtes plus faciles à défendre, et que leur histoire générale nous est mieux connue que celle des Arabes". Aggiunge inoltre denunciando i numerosi atti di pirateria cristiana: "Les témoignages des Chrétiens accusent eux-mémes tout le mal qu'on du faire leur propres pirates"; cfr. L. M. MAS LATRIE, Traités de paix et de commerce, cit., I, pp. 261; 233-234.
} 
tero la conquista portoghese sia in quelli che seguirono si deve ravvisare specialmente nella pirateria e nella corsa cristiana.

Dopo la presa di Ceuta l'attività corsara portoghese determinerà nell'estremo Mediterraneo occidentale un profondo cambiamento in quanto sostituirà, ad assalti pirateschi tra gli stati cristiani, le azioni dei portoghesi contro i mercanti musulmani che transitavano in quel mare per raggiungere località del Magreb e della costa atlantica. Una sia pur modesta vivacizzazione dell'attività della corsa musulmana è registrata qualche anno più tarji quando si verificherà la forte reazione contro Ceuta ${ }^{88}$.

Nei decenni che seguirono, João I riuscirà, malgrado la sconfitta di Tangeri del 1431, nel suo intento di inserirsi nel Mediterraneo tra le grandi potenze cristian e e di diminuire il loro potere economico e politico sul Magreb occidentale. Nel litorale dell' Africa atlantica le razzie, gia frequenti all'indomani dell'insediamento a Ceuta, saranno portate avanti fino a metà del secolo 89 .

Le modalità del contatto con le popolazioni africane si manterranno inalterate: gli assalti al litorale atlantico porteranno alla cattura di un numero rilevante di uomini che, come bestie ${ }^{00}$, saranno caricati forzatamente sui vascelli; l'unica novità e costituita dal tratto di costa che verrà esplorato proseguendo in direzione sud.

Ceuta portoghese si deve quindi considerare un avvenimento di grande rilievo per l'impulso che impresse alle imprese del Portogallo sul mare: l'evento darà l'avvio infatti alla realizzazione dei due importanti programmi che avranno luogo durante il corso del XV secolo, gli insediamenti portoghesi nel Magreb mediterraneo ed atlantico e l'esplorazione della costa occidentale dell'Africa. La Bolla di crociata del papa Martino $\mathrm{V}$, del 4 aprile del 1418, riconoscendo implicitamente allo stato iberico "la piazza di Ceuta e le altre città e terre che il re João avrebbe preso ai sara-

\footnotetext{
${ }^{88} \mathrm{Nel} 1420$ si concluse un accordo tra l'emiro di Granada e l'ultimo governatore di Ceuta, Salah-ben Salch, che prevedeva il blocco marittimo di Ceuta. La citta fu sottoposta a tre attacchi e resistette grazie all'invio di una spedizione comandata dall'Infante Enrico; cfr. F. SUREDA BLANES, Abyla Herculana, cit., p. 201.

${ }^{80}$ Il divieto di Enrico il Navigatore di compiere razzie porterà a "non combattere più le popolazioni di quei paesi"; in luogo delle razzie instaurera lo scambio di mercanzie, il resgate; cfr. Th. MONOD, R. MAUNY et G. DUVAL, De la première découverte de la Guinée, recits par Dogo Gomes, (fin XVe siecle), in "Centro de estudios da Guinée portuguesa", Bissau, 1959, p. 24.

${ }^{90} \mathrm{Cfr}$. Th. MONOD, R. MAUny et G. Duval, De la première découverte de la Guinde, cit., p. 22.
} 
ceni", non solo legittimava l'impresa compiuta ma poneva le basi per il successivo sviluppo del dominio portoghese in Africa ${ }^{91}$ di cui la corsa, ratificata da motivi religiosi, costituiva uno dei principali mezzi.

\section{RÉSUMÉ}

Dans les années qui précedent la conquête de Ceuta (1415) les nombreux événements de piraterie et de course dans la Mediterranée occidentale et dans le Détroit de Gibilterre provoquaient une condition de grande difficulté pour les trafiques avec le Maghrib occidentale et l'Emirat de Grenade. Les vaisseaux aragonaises que commerciaient dans les ports maghrebins, surtout en or, sont particulierement frappés par les assauts et les déprédements, les auteurs desquels étaient des autres marineries chrétiennes. Lettres adressées au roi d'Aragon, Fernando I, dénoncent d'une façon très preoccupée, une telle action qui aurait pu porter aussi à la fin des mêmes trafics.

En parallele, autre genre de documentation fait penser que la piraterie musulmane n'avait pas eu, en cet endroit et en ce période, une égale incidence. Les ostilités portugaises contre "l'infidele", (selon les chroniques de Zurara la "Crónica da tomada de Ceuta" et la "Crónica do Conde D. Pedro de Menezes"), plutot que contre la piraterie musulmane semblent être adressées, d'une partie à la conquête territoriale de la ville maghrebine, et donc au controle de la navigation musulmane et chrétienne dans le Détroit, et d'autre partie a l'anéantissement des trafiques commerciaux des musulmanes avec des attaques constants et systémathiques de leurs navires.

\section{SUMMARY}

In the years that preceded the Portuguese conquest of Ceuta (1415) frequent cases of piracy and privateering in the Western Mediterranean and

\footnotetext{
${ }^{91}$ Monumerta Henticina, cit., vol. II, pp. 283-284.
} 
in the Straits of Gibraltar created severe difficulties to sea traffic with the Western Maghreb and the Granada Emirate. The Aragonese merchant ships that traveled to Maghreb sea ports especially for gold supply were particularly hit by such assaults and robberies carried out by other Christian navies. Letters to the Aragonese sovereign, Fernando I, worriedly denounce this situation that might have destroyed the traffic completely

At the same time, taking into account other documents, we are led to presume that in this area and in this period Muslim piracy had a lesser impact. Hence rather than being aimed against Muslim piracy, the Portuguese struggle against the "infidels" (as seen in Zurara chronicles, the "Crónica da tomada de Ceuta" and the "Crónica do Conde D. Pedro de Menezes") seems to be mostly directed on one side toward the territorial conquest of the Maghrebian harbours and, therefore, toward controlling Muslim and Christian navigation in the Straits, and on the other side toward the destruction of Muslim commercial traffic through systematic attacks against Muslim ships. 